\title{
Celiac Disease Autoimmunity
}

\author{
Miguel Ángel López Casado ${ }^{1} \cdot$ Pedro Lorite $^{2} \cdot$ Candelaria Ponce de León ${ }^{2} \cdot$ Teresa Palomeque $^{2}$. \\ Maria Isabel Torres ${ }^{2} \mathbb{D}$
}

Received: 17 October 2017 / Accepted: 6 July 2018 / Published online: 25 August 2018

(C) L. Hirszfeld Institute of Immunology and Experimental Therapy, Wroclaw, Poland 2018

\begin{abstract}
Celiac disease is an autoimmune condition triggered by the ingestion of gluten, the protein fraction of wheat, barley and rye. It is not simply an intestinal disease; it is multifactorial caused by many different genetic factors acting together with non-genetic causes. Similar to other autoimmune diseases, celiac disease is a polygenic disorder for which the major histocompatibility complex locus is the most important genetic factor, and is the result of an immune response to self-antigens leading to tissue destruction and the autoantibodies production. Celiac disease exemplifies how an illness can have autoimmune-like features having to be driven by exogenous antigen and how can be reasonably considered as a model of organ-specific autoimmunity.
\end{abstract}

Keywords Celiac disease $\cdot$ Autoimmunity $\cdot$ Immune tolerance $\cdot$ Autoantibodies

\section{Celiac Disease as an Autoimmune Disorder}

Celiac disease $(\mathrm{CD})$ is one of the best-understood immunerelated diseases. $C D$ is frequent, with a prevalence of approximately $1: 100$, and it occurs selectively in individuals expressing HLA-DQ2 or HLA-DQ8. The prevalence of CD is underestimated, as not all cases of $\mathrm{CD}$ are symptomatic and thus go undiagnosed (Catassi and Fasano 2008) Celiac disease has many features in common with autoimmune disease.

In $\mathrm{CD}$, there is a massive, pro-inflammatory and pathogenic immune response towards certain parts of gluten and the intestinal tissue itself, resulting in structural changes. The pathogenesis of $\mathrm{CD}$ includes the potent and pathogenic $\mathrm{CD} 4^{+} \mathrm{T}$-cell response towards post-translationally modified gluten and the highly disease-specific B-cell responses towards deamidated gluten and the self-protein transglutaminase 2 (TG2) (Fig. 1).

It is important to consider how epithelial cell stress together with pro-inflammatory adaptive immunity causes cytotoxic T-cell-mediated tissue destruction in CD. The

Maria Isabel Torres

mitorres@ujaen.es

1 Department of Pediatrics Gastroenterology, Hospital Virgen de las Nieves, Granada, Spain

2 Department of Experimental Biology, University of Jaén, Jaén, Spain
HLA-DQ molecules predispose to disease by preferential presentation of gluten antigens to $\mathrm{CD} 4{ }^{+} \mathrm{T}$ cells (Bodd et al. 2012; Lundin et al. 1993; Torres et al. 2015). Although these predisposing HLA haplotypes are necessary for disease development, they are not sufficient, as they are highly prevalent in the general population.

The genetic predisposition to $\mathrm{CD}$ development has been studied by genome-wide association studies, which have found risk variants in the HLA region, especially HLADQ2.5, as well as HLA-DQ2.2 or HLA-DQ8 (Vader et al. 2003; Van Heel et al. 2007). Thirty-nine additional, nonHLA loci have been associated with CD (Hunt et al. 2008; Trynka et al. 2011). Of these genes, many are related to immunity, especially with T-cell and B-cell function. These non-HLA genes may be important determinants of disease susceptibility, as indirectly shown by the high disease concordance rate in monozygotic twins $(70 \%)$ compared with only 30\% in HLA-identical twins (Houlston and Ford 1996).

In general, the similarities between autoimmune diseases are multifaceted, with shared genetic, environmental and immunological factors that may explain the comorbidity. Novel theories have been put forth to explain the possible associations between autoimmune diseases, including the human gut microbiome's influence on the gut-immune system axis, new shared immunological markers, the discovery of common genetic factors, and environmental factors $(\mathrm{Wu}$ and $\mathrm{Wu}$ 2012; Zhernakova et al. 2009). 


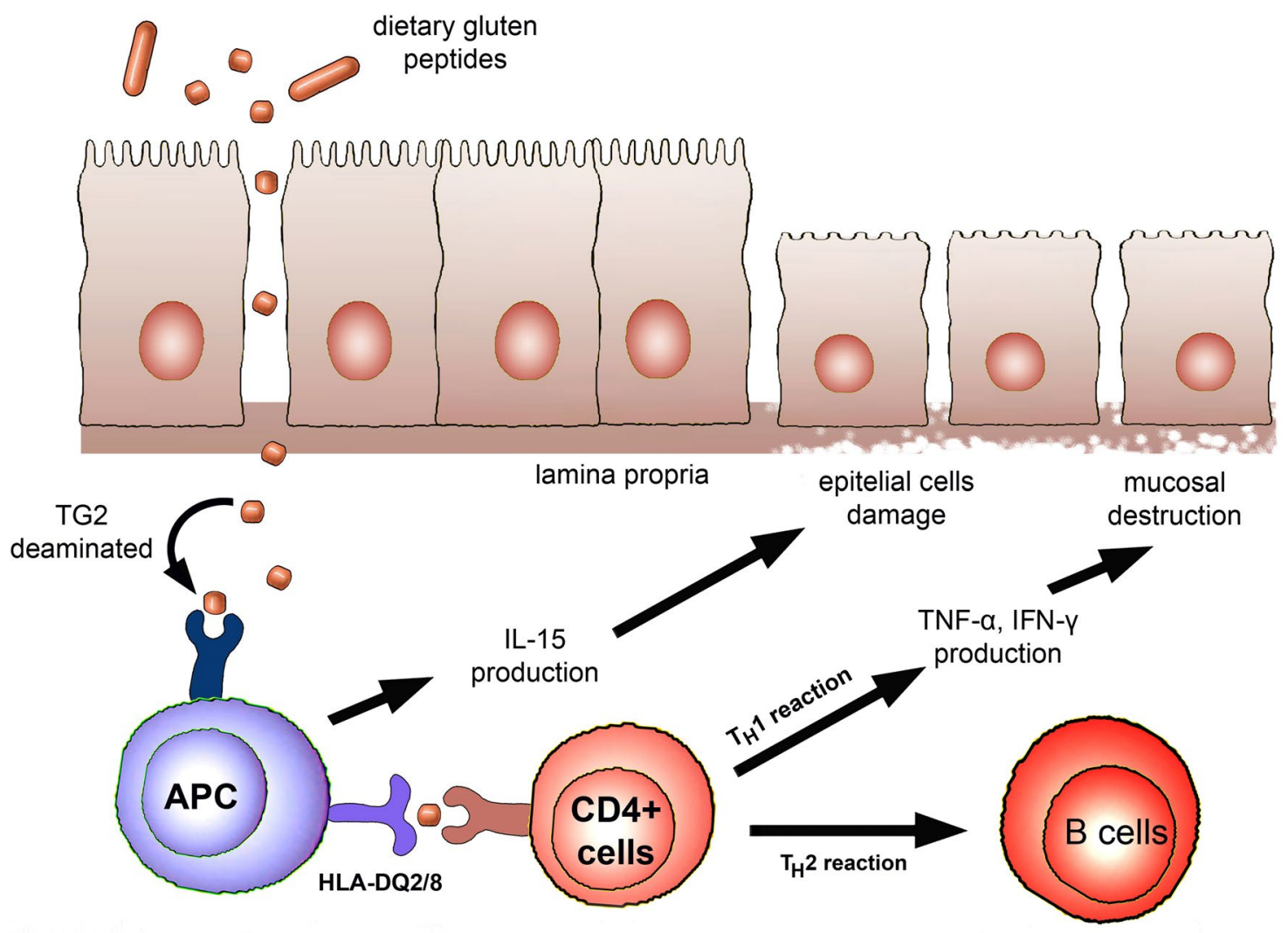

Fig. 1 Schematic representation of celiac disease pathogenesis. Gluten peptides can be transported across the intestinal epithelium. Deamidation by TG2 leads to the production of deamidated gluten that are taken up and presented by antigen-presenting cells (APCs) of HLADQ2/HLA-DQ8 molecules. This presentation leads to activated gluten-reactive $\mathrm{CD} 4^{+} \mathrm{Th} 1$ cells that produce high levels of proinflammatory cytokines, with aTh1 cytokine pattern dominated by

$\mathrm{CD}$ is an excellent model for studying the contribution of genetic factors to immune-related disorders, because (1) the environmental triggering factor is known (gluten); (2) as in other autoimmune diseases, specific HLA types (HLADQA1 and HLA-DQB1) are critically involved; (3) there is involvement of non-HLA disease-susceptibility loci, many of which are shared with other autoimmune diseases; (4) there is an elevated incidence of other immune-related diseases both in family members and in CD individuals; and (5) both the innate and the adaptive immune responses play a role in CD (Kumar et al. 2012; Zhernakova et al. 2009).

\section{Autoimmune Features of Celiac Disease}

Celiac disease is a unique autoimmune disorder in that the key genetic components (HLA class II genes DQ2 and/or DQ8) are present in almost all patients, the autoantigen is known (tissue transglutaminase), and, most importantly, the environmental trigger is known (gluten) (Lundin et al. 1993).
IFN- $\gamma$. Activated $\mathrm{CD} 4^{+} \mathrm{T}$ cells drive the activation and clonal expansion of B cells, which differentiate into plasma cells and produce antigliadin and anti-TG antibodies. Gluten peptides induce epithelial and APC cells to secrete IL-15, resulting in an increase in the number of IELs. IFN- $\gamma$ production, and stimulating cytotoxic effects on epithelial cells (Torres et al. 2015). Re-drawn of Sollid and Jabri (2013)

Gluten is not a pathogen and should not be perceived as immunogenic. Some undigested gluten peptides from Triticum species, mainly the 33-mer (LQLQPFPQPQLPYPQPQLPYPQPQLPYPQPQPF，P57-P89, from $\alpha-2$ gliadin) and the 25-mer (LGQQQPFPPQQPYPQPQPFPSQQPY, P31-P55 from $\alpha$-gliadin), have cytotoxic activity or immune-mediated activity (Ciccocioppo et al. 2005). Human TG2 deamidates the 33-mer peptide, increasing its immunogenicity. This enzyme converts glutamine residues into glutamic acid, which results in higher affinity of the peptides to HLA-DQ2/DQ8 molecules and subsequent induction of gluten-specific $\mathrm{CD} 4^{+} \mathrm{T}$-cell responses in the intestine of $\mathrm{CD}$ patients due to a complex interplay between innate and adaptative immune responses to ingested gluten (Barone et al. 2014; Jabri et al. 2005). The adaptative immune response to gluten appears to act in synergy with epithelial cell stress to allow intraepithelial $\mathrm{T}$ cells to induce villous atrophy in CD patients.

Gluten peptides, in contrast to other food peptides, are excellent substrates for TG2, and the gluten peptides with 
multiple B- and T-cell epitopes can be added onto the surface of multimeric complexes of TG2. $\mathrm{CD}^{+}$gluten-specific $\mathrm{T}$ cells provide help to activate $\mathrm{B}$ cells to produce antigluten and anti-TG2 antibodies in the plasma cells, which may serve as primary antigen-presenting cells (Lundin et al. 1993; Molberg et al. 1997). This could explain why hypersensitivity to gluten, but not to other food antigens, is established. It is important to understand how gluten-specific HLA-DQ-restricted T cells become pro-inflammatory and why this occurs only in CD patients and not in healthy patients.

It is believed that the interferon (IFN)- $\gamma$ production from these gluten-specific T cells may be the main cause of mucosal intestinal lesions (Mazzarella et al. 2008). Furthermore, shifts in intestinal permeability, secondary to changes in tight junctions or in food-antigen processing, have been associated with a loss of gluten tolerance (Lerner and Matthias 2015a).

\section{Autoantibodies}

TG2 is a deamidating enzyme that can increase the immunostimulatory effect of gluten and is a target autoantigen in the immune response (Di Sabatino et al. 2012). Autoantibodies to TG2 are usually used for diagnosis, showing high CD specificity and sensitivity (Sollid and Jabri 2011). The celiac disease-specific TG2-targeted autoantibodies are deposited in the small-bowel mucosa as well as in other tissues, and interestingly, extra-intestinal manifestations of the disease involving these particular tissues have been reported (Lindfors et al. 2010). As the TG2-targeted autoantibodies have experimentally been shown to modulate the function of different cell types in vitro similarly to what has been reported to occur in untreated celiac disease, they could constitute an important contribution to disease progression. So, subjects with negative serum TG2-specific antibodies still seem to produce these antibodies locally, as reflected by smallintestine deposits (Maglio et al. 2011). Antibodies to TG2 include $\operatorname{Ig} \mathrm{A}$ and $\mathrm{IgG}$ isotypes. IgA antibodies are more specific than IgG antibodies, and they are produced primarily in the mucosa of the intestine (Marzari et al. 2001). TG2 is involved in the formation of active transforming growth factor (TGF) $-\beta$ by the crosslinking of the TGF- $\beta$ binding protein and participates in the motility, as well as attachment, of fibroblasts and monocytes through interactions with fibronectin and integrins, causing $\mathrm{CD}$ villous atrophy (Akimov and Belkin 2001). Anti-TG2 IgA deposits are detectable in intestinal tissue before the development of overt $\mathrm{CD}$, suggesting that antibody production occurs early in the disease alongside the gluten-specific T-cell response.

Anti-TG2 antibodies may play a part in certain non-intestinal symptoms of CD by interacting with TG2, in addition to cross-reacting with other transglutaminases. In this sense, deposits of anti-transglutaminase antibody have been detected in the brainstem and cerebellum of a patient showing cerebellar ataxia and gluten sensitivity and in certain idiopathic, neurological, and psychiatric disorders (Hadjivassiliou et al. 2006). In addition, anti-gliadin antibodies reportedly bind to neural cells and cross-react specifically with synapsin I (Alaedini et al. 2007). Immune reactivity to other autoantigens, including transglutaminase 3 , actin, ganglioside, collagen, calreticulin and zonulin, has also been reported in CD (Alaedini and Green 2008). The clinical significance of these antibodies is not known, although some may be associated with specific clinical presentations or extra-intestinal manifestations of celiac disease.

\section{Autoreactive Intraepithelial Lymphocytes}

Dysregulated activation of intraepithelial lymphocytes (IELs) is a characteristic of CD that is implicated in the development of villous atrophy and epithelial cell injury. IELs constitute a population of antigen presentation like "innate" $\mathrm{T}$ cells that reside between enterocytes in the intestinal epithelium and can be activated by innate signals, acquiring a natural killer-like phenotype and cytotoxic effector functions (Jabri et al. 2000)..

$\mathrm{CD}$ is characterized (1) by the presence of gluten-specific $\mathrm{CD} 4^{+} \mathrm{T}$ cells in the lamina propria and (2) by a prominent intraepithelial T-cell infiltration in the epithelial layer that promotes the development of small-intestine inflammation. The exact role of $\mathrm{CD}^{+} \mathrm{T}$ cells in celiac disease is still not clear, so the excessive production of IFN- $\gamma$ may enhance HLA-E and MHC-I related chain (MIC) proteins expression by intestinal epithelial cells and promote cytotoxic responses by $\mathrm{CD} 8 \alpha \beta^{+}$- and/or CD $4^{+} \mathrm{TCR} \alpha \beta^{+}$-induced IELs through the innate CD94-NKG2D pathway (Sollid 2000). The upregulation of these NKG2 receptors seems to be driven by interleukin (IL)-15, which is expressed by CD enterocytes (Mention et al. 2003; Roberts et al. 2001). IL-15 seems to play a critical role in the expansion of IELs and in the induction of MIC molecules on intestinal epithelial cells (Hü et al. 2004).

IELs bearing the $\gamma \delta$ T-cell receptor (TCR) are more abundant in the small intestinal mucosa of patients with $\mathrm{CD}$ compared with healthy individuals. Increased numbers of $\mathrm{TCR} \gamma \delta^{+}$IELs have also been observed in the small intestinal mucosa of $\mathrm{CD}$ patients with latent $\mathrm{CD}$, or those on glutenfree diet (GFD) and in some first-degree relatives of CD patients with HLA-DQ2 (Holm et al. 1992). Additionally, the increase of intraepithelial $\mathrm{TCR} \gamma \delta^{+} \mathrm{T}$ cells is more pronounced in children than in adults with active CD (Savilahti et al. 1997). It has been proposed that the intraepithelial infiltration of TCR $\gamma \delta^{+} \mathrm{T}$ cells could be used as a diagnostic marker to identify early stage CD (Jarvinen et al. 2003) or to predict the risk of $\mathrm{CD}$ development among at-risk subjects 
with positive CD-specific autoantibodies and normal intestinal biopsy (Paparo et al. 2005).

Similar to gluten-reactive T cells, gluten-specific B cells preferentially recognize deamidated gluten peptides (Iversen et al. 2015). Close interactions between $\mathrm{CD}^{+}{ }^{+} \mathrm{T}$ cells and $\mathrm{B}$ cells may be important for amplifying the inflammatory response, as B cells can present antigen to T cells and they in turn can provide help for autoantibody production. Glutenreactive $\mathrm{CD} 4^{+} \mathrm{T}$ cells provide help to both TG2-reactive $\mathrm{B}$ cells and deamidated gluten-reactive B cells. TG2-gluten complexes are internalized by TG2-reactive B cells through B-cell receptor-mediated endocytosis. After internalization, deamidated gluten peptides can be released and can bind to HLA-DQ2 or HLA-DQ8 to be presented to T cells. Cooperation between gluten-reactive T-cell and B-cell results in activation of both the T cells and B cells, leading the B cells to differentiate into antibody-producing plasma cells and the $\mathrm{T}$ cells to proliferate and clonally expand.

\section{Genetics of Celiac Disease}

The genetics of autoimmune diseases include the intracellular signaling that drives the activation of $\mathrm{T}$ and $\mathrm{B}$ cells, signaling by cytokines and their receptors, and pathways that mediate innate immunity and microbial responses, such as Toll-like receptors and nucleotide-binding oligomerization domain receptors (Sollid and Jabri 2013).

Similar to other autoimmune diseases, celiac disease is a polygenic disorder for which the MHC locus is the single most important genetic factor. The MHC locus accounts for $40-50 \%$ of the genetic variance in the disease. The great majority of patients carry a particular variant of HLA-DQ2 (DQA1*05:01, DQB1*02:01; also known as DQ2.5). Those who are not DQ2.5 are almost all HLA-DQ8 (DQA $1 * 03, \mathrm{DQB} 1 * 03: 02$ ) or carry another variant of HLADQ2 (DQA1*02:01, DQB1:02:02; also known as DQ2.2) (Megiorni and Pizzuti 2012; Smyth et al. 2008; Sollid and Lie 2005). HLA can be considered a necessary, but not sufficient, factor for disease development. HLA testing is much used in the clinic to exclude the diagnosis of celiac disease.

Many of the susceptibility loci for celiac disease are shared with those for other autoimmune diseases, such as type 1 diabetes (T1D) and rheumatoid arthritis (RA) (Gutierrez-Achury et al. 2011; Zhernakova et al. 2011), most of which encode genes involved in inflammatory and immune responses (Kumar et al. 2012). Some of them may act as regulators of proliferation and activity of $\mathrm{T}$ lymphocytes (CTLA-4, ICOSLG, and IL18RAP). Other genes have also been implicated in the activity of nuclear factor kappa B (REL, UBE2LE) and in signaling processes (SOCS1, $\mathrm{SH} 2 \mathrm{~B} 3$ ) or in more than one function, as in the activities of T lymphocytes and cytokines (IL2, IL21, ILI2A and IL23R)
(Anaya et al. 2012; Larizza et al. 2012). The HLA locus still presents the most important association in individuals affected by more than one autoimmune disorder (double autoimmunity), and that carry more of the genetic risk markers that are shared between the two diseases independently. In this sense, the CTLA4 and IL2RA loci were more strongly associated with double autoimmunity than with either T1D or CD alone. HLA analyses indicated that the T1D high-risk genotype, $D Q 2.5 / D Q 8$, provided the highest risk for developing double autoimmunity.

The HLA and non-HLA loci can be used as stratification factors in the construction of risk models to predict double autoimmunity appearance and for pathway enrichment analysis to enhance our understanding of the pathophysiology involved in the development of both autoimmune diseases.

\section{Environmental Factors in Celiac Disease}

Gluten is the environmental factor required to trigger the disease, but other factors may be involved in a model of a complex multifactorial disease. The intestinal infections, the amount and quality of ingested gluten, the composition of intestinal microbiota, and infant-feeding practices are all possible triggers of the switch from tolerance to an immune response to gluten (Prescott et al. 2008). In this sense, two randomized controlled trials have been performed to clarify the relationship between the age at which gluten is introduced to a child's diet and the risk of $\mathrm{CD}$, showing that timing of gluten introduction does not modify the risk of $\mathrm{CD}$ (Lionetti et al. 2014; Vriezinga et al. 2014). Also showed that breastfeeding duration or breastfeeding during gluten introduction have no effect on the risk of CD.

The study of Bouziat et al. (2017) provides support for the concept that viruses can disrupt intestinal immune homeostasis and initiate loss of oral tolerance and Th1 immunity to dietary antigen. The authors propose that viruses elicit proinflammatory immune responses to dietary antigen altering immune homeostasis and in particular outfit dendritic cells (DCs) with pro-inflammatory properties at sites, where oral tolerance is induced (Bouziat et al. 2017). Although reovirus infections may trigger development of Th1 immunity to gluten as well as activation of TG2, additional events will be required for induction of anti-TG2 antibodies and villous atrophy.

Many of the implicated environmental factors may act by altering the composition of the microbiome (Verdu et al. 2015). Epidemiological data support an association between dysbiosis and increased risk of CD but there is little understanding of how it might influence gluten-specific immunity in vivo. In vitro data supports the influence of microbes on immune responses to gluten, including roles in modifying $\mathrm{T}$ regulatory cells induction, epithelial cell stress and IEL 
activation, phenotypic and functional maturation of DCs and pro-inflammatory cytokine production (Verdu et al. 2015).

\section{The Human Microbiome as a Modulator of Autoimmunity in Celiac Disease}

The high increase in the incidence of autoimmune disorders cannot be explained only by genetic drift and is thought to be the result of changes in the environment.

The microbiota plays an important role in immune maturation and homeostasis; alterations in microbial composition or colonization may influence intestinal homeostasis and, consequently, immune responses to food antigens.

$\mathrm{CD}$ has been linked to alterations in microbial composition (named intestinal dysbiosis) that could promote the disease onset and progression, as for other autoimmune disorders, such as T1D, multiple sclerosis and RA (Edwards 2008; Leirisalo-Repo 2005). It is unknown whether dysbiosis is a disease-promoting factor.

Differences in microbial metabolites between faecal samples of $\mathrm{CD}$ patients and healthy controls point to a functional role of the microbiota in the pathogenesis of CD (Di Cagno et al. 2009; Tjellstrom et al. 2005). A significantly higher number of Gram-negative and potentially pro-inflammatory bacteria were found to be associated with the symptomatic presentation of CD (Nadal et al. 2007). The unbalanced microbiota in children with untreated CD seems only partially restored after long-term treatment with a gluten-free diet (Collado et al. 2008; Sanz et al. 2007).

Little is known about the association between dysbiosis and gluten-specific T-cell responses. The functional relevance of these associations in $\mathrm{CD}$ remains unclear. The dysbiosis in $\mathrm{CD}$ is hallmarked by an increase in gram-negative and Bacteroides species and by a decrease in Bifidobacteria and Lactobacilli (Cheng et al. 2013). As the intestinal microbiota is able to modulate the cytokine environment, an unfavourable microbiota could amplify the immune response to gliadin in individuals with CD. Dysbiosis could represent an important trigger in $\mathrm{CD}$ pathogenesis, along with genetic (HLA haplotypes) and environmental (antibiotic administration, mode of delivery, and breastfeeding) factors (Losurdo et al. 2016).

Many studies have shown the presence of bacteria-derived proteolytic activities with the ability to hydrolyse gluten peptides in saliva, the duodenum and faeces (Caminero et al. 2012; Helmerhorst et al. 2010). Lactobacillus strains have the ability to completely hydrolyse the 33 -mer peptide. However, the degradation products generated after the 33-mer hydrolysis are unknown and could also be highly immunogenic. It is fundamental to test these degradation products to determine whether the immunogenic epitopes are destroyed (Caminero et al. 2016). The opportunistic pathogens and core gut commensals generate distinct breakdown patterns of gluten with increased or decreased immunogenicity that could influence autoimmune risk. Gluten proteins are resistant to mammalian protease degradation but are good substrates for bacterial metabolic activity (Caminero et al. 2016). Proteases produced by environmental microorganisms have been proposed as pharmacologic therapy in $\mathrm{CD}$ (Lahdeaho et al. 2014; Tack et al. 2013).

\section{Gluten-Free Diet and Autoimmune Disorders}

It has been suggested that the intestinal-barrier dysfunction associated with undiagnosed CD might promote the onset of other autoimmune disorders by increasing the intestinal permeability to certain triggers (Shan et al. 2004). Diet intervention is a multifactorial approach, because gluten may affect not only the immune system and the gut microbiota but also other organs, such as the pancreas and liver. Ventura et al. (2012) were the first to report gluten-associated autoimmunity. The longer the duration of exposure to gluten before diagnosis of $\mathrm{CD}$ is, the higher the risk of developing autoimmune disorders later in life.

Epidemiological evidence suggests that a GFD may have a positive effect in the protection against T1D in humans with CD (Cosnes et al. 2008). However, the evidence is conflicting, as other studies found no protection (Viljamaa et al. 2005). The importance of gluten in T1D is highlighted by cohort studies finding that early introduction of gluten in the diet (before 3 months of age) increases the prevalence of diabetes autoantibodies in high-risk individuals (Ziegler et al. 2003). One of these studies showed signs of improved insulin sensitivity and insulin secretion in the GFD group compared to the group on a normal diet (Pastore et al. 2003).

Kaukinen et al. (2002) have described CD patients with severe liver injury displaying improvement after a GFD. One of these patients had early cirrhosis, and institution of a GFD led to the disappearance of ascites, although the micronodular cirrhosis persisted.

A later onset of $\mathrm{CD}$ is related to greater intestinal-barrier integrity and diminishing antigen triggers in the case of several autoimmune diseases. More large-scale prospective studies would be helpful to elucidate the way in which CD is related to other autoimmune conditions to clarify the possible influence that a GFD exerts on these conditions.

\section{Others Autoimmune Disorders Associated with Celiac Disease}

CD tends to coexist with several autoimmune diseases, including T1D, autoimmune thyroiditis, inflammatory bowel disease, rheumatoid arthritis, connective tissue disorders 
Table 1 Celiac disease and associated autoimmune disorders

\begin{tabular}{ll}
\hline Liver diseases & Connective tissue diseases \\
Primary biliary cirrhosis & Rheumatoid arthritis \\
Autoimmune hepatitis & Sjogren's syndrome \\
Primary sclerosing hepatitis & Systemic lupus erythema- \\
tosus
\end{tabular}

and psoriasis between others (Birkenfeld et al. 2009; Lerner and Matthias 2015a, b; Viljamaa et al. 2005) (Table 1). The prevalence of co-existing autoimmune disease is estimated to be 3-10 times higher in CD patients than in the general population (Kakleas et al. 2015; Sategna Guidetti et al. 2001). In CD patients, a diagnosis of the disease early in life and a positive family history of autoimmunity are risk factors for developing other autoimmune diseases, while a GFD has a protective effect (Collin et al. 1994). More than $60 \%$ of CD-associated susceptibility loci are shared with at least one other autoimmune condition, such as T1D and RA (Rossi and Bot 2011), suggesting common pathogenic mechanisms. In particular, the recognition of peptides by HLA molecules, post-translational modifications of selfantigens (PTMs) required for optimal peptide binding, and immune mechanisms leading to tissue damage have been found. PTMs allow the generation of neo-self epitopes in the development of autoimmunity (Doyle and Mamula 2005). PTMs can occur spontaneously or arise by enzymatic modification, altering the protein structure, biological functions and. modifications of the proteolytic degradation. PTMs of self-proteins to which the immune system has not developed tolerance, include enzyme-dependent glycosylation, deamidation, citrullination, iodination, phosphorylation, methylation or chemical modifications such as disulfide bridge formation, oxidative modification or nitration, and many others (Arentz-Hansen et al. 2000; Collado et al. 2013).

Individuals affected by more than one autoimmune disorder may have an immune response more disturbed than those with only one disease. There may be an overlap in their aetiology due to shared genetic risk factors (Zhernakova et al. 2009) or due to synergistic effects of the genes involved in each disease separately (Cotsapas et al. 2011). The larger percentage of individuals developing more than one autoimmune disorder than expected suggests that common genetic loci and common biological pathways are involved in their pathogenesis. Regular screening in patients with one autoimmune disease for autoantibodies of other autoimmune diseases will be important for the clinical care of these patients and may provide some insights into the disease pathogenesis.

Acknowledgements This work was supported by Junta de Andalucia throughout the programme "Ayudas a grupos investigación BIO-220".

\section{References}

Akimov SS, Belkin AM (2001) Cell surface tissue transglutaminase is involved in adhesion and migration of monocytic cells on fibronectin. Blood 98:1567-1576

Alaedini A, Green PH (2008) Autoantibodies in celiac disease. Autoimmunity 41:19-26

Alaedini A, Okamoto H, Briani C et al (2007) Immune cross-reactivity in celiac disease: anti-gliadin antibodies bind to neuronal synapsin I. J Immunol 178:6590-6595

Anaya JM, Kim-Howard X, Prahalad S et al (2012) Evaluation of genetic association between an ITGAM non-synonymous SNP (rs1143679) and multiple autoimmune diseases. Autoimmun Rev 11:276-280

Arentz-Hansen H, Körner R, Molberg O et al (2000) The intestinal T cell response to alpha-gliadin in adult celiac disease is focused on a single deamidated glutamine targeted by tissue transglutaminase. J Exp Med 191:603-612

Barone MV, Troncone R, Auricchio S (2014) Gliadin peptides as triggers of the proliferative and stress/innate immune response of the celiac small intestinal mucosa. Int J Mol Sci 15:20518-20537

Birkenfeld S, Dreiher J, Weitzman D et al (2009) Coeliac disease associated with psoriasis. Br J Dermatol 161:1331-1334

Bodd M, Kim CY, Lundin KE et al (2012) T-cell response to gluten inpatients with HLA-DQ2.2 reveals requirement of peptide-MHC stability in celiac disease. Gastroenterology 142:552-561

Bouziat R, Hinterleitner R, Brown JJ et al (2017) Reovirus infection triggers inflammatory responses to dietary antigens and development of celiac disease. Science 356:44-50

Caminero A, Nistal E, Arias L et al (2012) A gluten metabolism study in healthy individuals shows the presence of faecal glutenasic activity. Eur J Nutr 51:293-299

Caminero A, Galipeau HJ, McCarville JL et al (2016) Duodenal bacteria from patients with celiac disease and healthy subjects distinctly affect gluten breakdown and immunogenicity. Gastroenterology 151:670-683

Catassi C, Fasano A (2008) Celiac disease. Curr Opin Gastroenterol 24:687-691

Cheng J, Kalliomäki M, Heilig HG et al (2013) Duodenal microbiota composition and mucosal homeostasis in pediatric celiac disease. BMC Gastroenterol 13:113

Ciccocioppo R, Di Sabatino A, Corazza GR (2005) The immune recognition of gluten in coeliac disease. Clin Exp Immunol 140:408-416 
Collado MC, Donat E, Ribes-Koninckx C et al (2008) Imbalances in faecal and duodenal Bifidobacterium species composition in active and non-active coeliac disease. BMC Microbiol 8:232

Collado JA, Guitart C, Ciudad MT et al (2013) The repertoires of peptides presented by MHC-II in the thymus and in peripheral tissue: a clue for autoimmunity? Front Immunol 4:442

Collin P, Reunala T, Pukkala E et al (1994) Coeliac disease-associated disorders and survival. Gut 35:1215-1218

Cosnes J, Cellier C, Viola S et al (2008) Incidence of autoimmune diseases in celiac disease: protective effect of the gluten-free diet. Clin Gastroenterol Hepatol 6:753-758

Cotsapas C, Voight BF, Rossin E et al (2011) Pervasive sharing of genetic effects in autoimmune disease. PLoS Genet 7:e1002254

Di Cagno R, Rizzello CG, Gagliardi F et al (2009) Different fecal microbiotas and volatile organic compounds in treated and untreated children with celiac disease. Appl Environ Microbiol 75:3963-3971

Di Sabatino A, Vanoli A, Giuffrida P et al (2012) The function of tissue transglutaminase in celiac disease. Autoimmun Rev 11:746-753

Doyle HA, Mamula MJ (2005) Posttranslational modifications of selfantigens. Ann NY Acad Sci 1050:1-9

Edwards CJ (2008) Commensal gut bacteria and the etiopathogenesis of rheumatoid arthritis. J Rheumatol 35:1477-14797

Gutierrez-Achury J, Coutinho de Almeida R, Wijmenga C (2011) Shared genetics in coeliac disease and other immune-mediated diseases. J Intern Med 269:591-603

Hadjivassiliou M, Mäki M, Sanders DS et al (2006) Autoantibody targeting of brain and intestinal transglutaminase in gluten ataxia. Neurology 66:373-377

Helmerhorst EJ, Zamakhchari M, Schuppan D et al (2010) Discovery of a novel and rich source of gluten-degrading microbial enzymes in the oral cavity. PLoS One 5:e13264

Holm K, Maki M, Savilahti E et al (1992) Intraepithelial gamma delta T-cell-receptor lymphocytes and genetic susceptibility to coeliac disease. Lancet 339:1500-1503

Houlston RS, Ford D (1996) Genetics of coeliac disease. QJM 89:737-743

Hü ES, Mention JJ, Monteiro RC et al (2004) A direct role for NKG2D/ MICA interaction in villous atrophy during celiac disease. Immunity 21:367-377

Hunt KA, Zhernakova A, Turner G et al (2008) Newly identified genetic risk variants for celiac disease related to the immune response. Nat Genet 40:395-402

Iversen R, Du Pré MF, Di Niro R et al (2015) Igs as substrates for transglutaminase 2: implications for autoantibody production in celiac disease. J Immunol 195:5159-5168

Jabri B, De Serre NP, Cellier C et al (2000) Selective expansion of intraepithelial lymphocytes expressing the HLA-E-specific natural killer receptor CD94 in celiac disease. Gastroenterology 118:867-879

Jabri B, Kasarda DD, Green PH (2005) Innate and adaptive immunity: the yin and yang of celiac disease. Immunol Rev 206:219-231

Jarvinen TT, Kaukinen K, Laurila K et al (2003) Intraepithelial lymphocytes in celiac disease. Am J Gastroenterol 98:1332-1337

Kakleas K, Soldatou A, Karachaliou F (2015) Associated autoimmune diseases in children and adolescents with type 1 diabetes mellitus (T1DM). Autoimmun Rev 14:781-797

Kaukinen K, Halme L, Collin P et al (2002) Celiac disease in patients with severe liver disease: Gluten free diet may reverse hepatic failure. Gastroenterology 122:881-888

Kumar V, Wijmenga C, Withoff S (2012) From genome-wide association studies to disease mechanisms: celiac disease as a model for autoimmune diseases. Semin Immunopathol 34:567-580

Lahdeaho ML, Kaukinen K, Laurila K et al (2014) Glutenase ALV003 attenuates gluten-induced mucosal injury in patients with celiac disease. Gastroenterology 146:1649-1658
Larizza D, Calcaterra V, Klersy C et al (2012) Common immunogenetic profile in children with multiple autoimmune diseases: the signature of HLA-DQ pleiotropic genes. Autoimmunity 45:470-475

Leirisalo-Repo M (2005) Early arthritis and infection early arthritis and infection. Curr Opin Rheumatol 17:433-439

Lerner A, Matthias T (2015a) Changes in intestinal tight junction permeability associated with industrial food additives explain the rising incidence of autoimmune disease. Autoimmun Rev 14:479-489

Lerner A, Matthias T (2015b) Rheumatoid arthritis-celiac disease relationship: joints get that gut feeling. Autoimmun Rev 14:1038-1047

Lindfors K, Mäki M, Kaukinen K (2010) Transglutaminase 2-targeted autoantibodies in celiac disease: pathogenetic players in addition to diagnostic tools? Autoimmun Rev 9:744-749

Lionetti E, Castellaneta S, Francavilla R et al (2014) Introduction of gluten, HLA status, and the risk of celiac disease in children. $\mathrm{N}$ Engl J Med 371:1295-1303

Losurdo G, Principi M, Iannone A et al (2016) The interaction between celiac disease and intestinal microbiota. J Clin Gastroenterol 50(Suppl 2):S145-S147

Lundin KE, Scott H, Hansen T et al (1993) Gliadin-specific, HLA$\mathrm{DQ}\left(1 * 05011^{*} 0201\right)$ restricted $\mathrm{T}$ cells isolated from the small intestinal mucosa of celiac disease patients. J Exp Med 178:187-196

Maglio M, Tosco A, Auricchio R et al (2011) Intestinal deposits of anti-tissue transglutaminase IgA in childhood celiac disease. Dig Liver Dis 43:604-608

Marzari R, Sblattero D, Florian F et al (2001) Molecular dissection of the tissue transglutaminase autoantibody response in celiac disease. J Immunol 166:4170-4176

Mazzarella G, Stefanile R, Camarca A et al (2008) Gliadin activates HLA class I-restricted $\mathrm{CD} 8^{+} \mathrm{T}$ cells in celiac disease intestinal mucosa and induces the enterocyte apoptosis. Gastroenterology 134:1017-1027

Megiorni F, Pizzuti A (2012) HLA-DQA1 and HLA-DQB1 in Celiac disease predisposition: practical implications of the HLA molecular typing. J Biomed Sci 19:88

Mention JJ, Ben Ahmed M, Begue B et al (2003) Interleukin 15: a key to disrupted intraepithelial lymphocyte homeostasis and lymphomagenesis in celiac disease. Gastroenterology 125:730-745

Molberg O, Kett K, Scott H et al (1997) Gliadin specific, HLA DQ2restricted $\mathrm{T}$ cells are commonly found in small intestinal biopsies from coeliac disease patients, but not from controls. Scand J Immunol 46:103-109

Nadal I, Donat E, Ribes-Koninckx C et al (2007) Imbalance in the composition of the duodenal microbiota of children with coeliac disease. J Med Microbiol 56(Pt 12):1669-1674

Paparo F, Petrone E, Tosco A et al (2005) Clinical, HLA, and small bowel immunohistochemical features of children with positive serum antiendomysium antibodies and architecturally normal small intestinal mucosa. Am J Gastroenterol 100:2294-2298

Pastore MR, Bazzigaluppi E, Belloni C et al (2003) Six months of gluten-free diet do not influence autoantibody titers, but improve insulin secretion in subjects at high risk for type 1 diabetes. J Clin Endocrinol Metab 88:162-165

Prescott SL, Smith P, Tang M et al (2008) The importance of early complementary feeding in the development of oral tolerance: concerns and controversies. Pediatr Allergy Immunol 19:375-380

Roberts AI, Lee L, Schwarz E et al (2001) NKG2D receptors induced by IL- 15 costimulate CD28-negative effector CTL in the tissue microenvironment. J Immunol 167:5527-5530

Rossi M, Bot A (2011) Celiac disease: progress towards diagnosis and definition of pathogenic mechanisms. Int Rev Immunol 30:183-184 
Sanz Y, Sanchez E, Marzotto M et al (2007) Differences in faecal bacterial communities in coeliac and healthy children as detected by PCR and denaturing gradient gel electrophoresis. FEMS Immunol Med Microbiol 51:562-568

Sategna Guidetti C, Solerio E, Scaglione N et al (2001) Duration of gluten exposure in adult coeliac disease does not correlate with the risk for autoimmune disorders. Gut 49:502-505

Savilahti E, Ormälä T, Arato A et al (1997) Density of gamma/delta $+\mathrm{T}$ cells in the jejunal epithelium of patients with coeliac disease and dermatitis herpetiformis is increased with age. Clin Exp Immunol 109:464-467

Shan L, Marti T, Sollid LM et al (2004) Comparative biochemical analysis of three bacterial prolyl endopeptidases: implications for coeliac sprue. Biochem J 383(Pt 2):311-318

Smyth DJ, Plagnol V, Walker NM et al (2008) Shared and distinct genetic variants in type 1 diabetes and celiac disease. $\mathrm{N}$ Engl $\mathrm{J}$ Med 359:2767-2777

Sollid LM (2000) Molecular basis of celiac disease. Annu Rev Immunol 18:53-81

Sollid LM, Jabri B (2011) Celiac disease and transglutaminase 2: a model for posttranslational modification of antigens and HLA association in the pathogenesis of autoimmune disorders. Curr Opin Immunol 23:732-738

Sollid LM, Jabri B (2013) Triggers and drivers of autoimmunity: lessons from coeliac disease. Nat Rev Immunol 13:294-302

Sollid LM, Lie BA (2005) Celiac disease genetics: current concepts and practical applications. Clin Gastroenterol Hepatol 3:843-851

Tack GJ, Van de Water JM, Bruins MJ et al (2013) Consumption of gluten with gluten-degrading enzyme by celiac patients: a pilotstudy. World J Gastroenterol 19:5837-5847

Tjellstrom B, Stenhammar L, Hogberg L et al (2005) Gut microflora associated characteristics in children with celiac disease. Am J Gastroenterol 100:2784-2788

Torres MI, Palomeque T, Lorite P (2015) Celiac disease and other autoimmune disorders. In: Chatzidionysiou K (ed) Autoimmunitypathogenesis, clinical aspects and therapy of specific autoimmune diseases. Intech, Croatia, pp 131-151
Trynka G, Hunt KA, Bockett NA et al (2011) Dense genotying identifies and localizes multiple common and rare variant association signals in celiac disease. Nat Genet 43:1193-1201

Vader W, Stepniak D, Kooy Y et al (2003) The HLA-DQ2 gene dose effect in celiac disease is directly related to the magnitude and breadth of gluten-specific T cell responses. Proc Natl Acad Sci USA 100:12390-12395

Van Heel DA, Franke L, Hunt KA et al (2007) A genome-wide association study for celiac disease identifies risk variants in the region harboring IL2 and IL21. Nat Genet 39:827-829

Ventura M, Turroni F, Motherway MO et al (2012) Host-microbe interactions that facilitate gut colonization by commensal bifidobacteria. Trends Microbiol 20:467-476

Verdu EF, Galipeau HJ, Jabri B (2015) Novel players in coeliac disease pathogenesis: role of the gut microbiota. Nat Rev Gastroenterol Hepatol 12:497-506

Viljamaa M, Kaukinen K, Huhtala H et al (2005) Coeliac disease, autoimmune diseases and gluten exposure. Scand J Gastroenterol 40:437-443

Vriezinga SL, Auricchio R, Bravi E et al (2014) Randomized feeding intervention in infants at high risk for celiac disease. N Engl J Med 371:1304-1315

Wu HJ, Wu E (2012) The role of gut microbiota in immune homeostasis and autoimmunity. Gut Microbes 3:4-14

Zhernakova A, van Diemen CC, Wijmenga C (2009) Detecting shared pathogenesis from the shared genetics of immune-related diseases. Nat Rev Genet 10:43-55

Zhernakova A, Stahl EA, Trynka G et al (2011) Meta-analysis of genome-wide association studies in celiac disease and rheumatoid arthritis identifies fourteen non-HLA shared loci. PLoS Genet 7:e1002004

Ziegler AG, Schmid S, Huber D et al (2003) Early infant feeding and risk of developing type 1 diabetes-associated autoantibodies. JAMA 290:1721-1728 